\title{
Germanica
}

\section{Epiphanie des Augenblicks. Zeit im Werk Thomas Glavinics}

Epiphanie de l'instant. Le temps dans les cuvres de Thomas Glavinic The epiphany of the moment. Time in Thomas Glavinic's works

\section{Felix Forsbach}

\section{OpenEdition}

Journals

Édition électronique

URL : http://journals.openedition.org/germanica/2700

DOI : $10.4000 /$ germanica. 2700

ISSN : 2107-0784

\section{Éditeur}

Université de Lille

Édition imprimée

Date de publication : 30 décembre 2014

Pagination : 175-188

ISBN : 9782913857346

ISSN : 0984-2632

\section{Référence électronique}

Felix Forsbach, « Epiphanie des Augenblicks. Zeit im Werk Thomas Glavinics », Germanica [Online], 55 | 2014, Online erschienen am: 30 Dezember 2016, abgerufen am 06 Oktober 2020. URL : http:// journals.openedition.org/germanica/2700 ; DOI : https://doi.org/10.4000/germanica.2700 


\title{
Epiphanie des Augenblicks. Zeit im Werk Thomas Glavinics.
}

\author{
Felix FORSBACH \\ Otto-Friedrich Universität Bamberg
}

„König der Zeitkapsel“1

Thomas Glavinc

\section{Gesellschaftstendenz: Beschleunigung}

Nicht erst seit dem es den kritischen Begriff der Entschleunigung gibt, um auf die Beschleunigung der Gesellschaft und des Lebens heute hinzuweisen, wurde in der Entwicklung der postmodernen Gesellschaft auf eine Beschleunigung als eine allgemeine Tendenz hingewiesen. Paul Virilio beschreibt dies prominent in seiner Dromologie - einer Wissenschaft, die sich mit der Geschwindigkeitszunahme auseinandersetzt. Im folgenden Aufsatz wird die Thematisierung von Zeit in der Roman-Trilogie ${ }^{2}$ des österreichischen Gegenwartsromanciers Thomas Glavinic behandelt.

1. - Thomas Glavinic, Das größere Wunder, München, Hanser, S. 404.

2. - Die Roman-Trilogie, die nicht als solche gekennzeichnet ist, umfasst die Romane Die Arbeit der Nacht, Das Leben der Wünsche und Das größere Wunder. Die Romane werden als Trilogie gesehen, weil sie alle einen gleichnamigen Erzähler Jonas haben und von einer Liebe zu Marie handeln. Außerdem werden in allen drei Texten ähnliche Themen aufgegriffen. Die Themen sind unter anderen Liebe, Glück, Tod, Medien, Träume und Zeit. 
Physikalisch ist eine Beschleunigung der Zeit unmöglich, vielmehr ist es eine Beschleunigung in der Zeit. Wenn Zeit als beschleunigt bezeichnet wird, liegt dies eher in der Wahrnehmung des Menschen begründet. Denn eine naturwissenschaftlich nachweisbare Beschleunigung von Zeit ist unmöglich. Ein großer Teil der philosophischen Auseinandersetzung mit Zeit erläutert diese vor der Annahme, dass sie linear verlaufe ${ }^{3}$. Daraus resultiert, dass es einzelne Punkte auf einer Linie ähnlich einem Zeitstrahl gibt. Diese einzelnen Punkte sind als isolierte Jetzt-Momente zu sehen. Die Zeit wird immer aus der Perspektive der Gegenwart, genauer eines Jetzt-Moments, in Vergangenes und Zukünftiges aufgeteilt. So tritt zur Kategorie der Zeit in den meisten philosophischen Auseinandersetzungen der Raum als zweite fundamentale phänomenologisch zu erforschende Kategorie hinzu. Der Standpunkt, von dem aus Zeit beobachtet wird, ist der des Menschen im Hier und Jetzt. Dieses Zusammendenken von Zeit und Raum kritisierte bereits Hegel $^{4}$ und es scheint mit der Multiplikation der Möglichkeiten in der Telekommunikationstechnik erst recht zu einem Lösen der Verbindung des Hier und Jetzt also des Raums von der Zeit gekommen zu sein. Virilio schreibt dazu:

„Auf der einen Seite die Echtzeit unseres unmittelbaren Tuns, bei der wir hier und jetzt handeln, und auf der anderen Seite die Echtzeit einer medialen Interaktivität, die das $>$ jetzt $<$ der Sendezeit der Fernsehsendung auf Kosten des $>$ Hier $<$ d.h. des Raums, in dem sich der Ort der Begegnung befindet, begünstigt" .

Dank der modernen Technik treten der Raum und die Zeit auseinander. Diese Idee ist auch bei Glavinic formuliert, wenn er in Das Leben der Wünsche schreibt, dass es egal sei „,[o]b es eine Live-Übertragung von einem anderen Kontinent war oder nur in den Nachbarort geschaltet wurde -immer wunderte es ihn ein wenig, dass diese andere Wirklichkeit möglich war"6. Von diesen anderen Möglichkeiten erfahren wir durch Live-Übertragungen im Fernsehen, Telefonate oder zahlreiche andere Telekommunikationsmedien. Für Virilio tritt an diesen Stellen das Hier und Jetzt auseinander.

Thomas Glavinic wiederum sieht es als Hier und Jetzt verschiedener Menschen und ihrer Wahrnehmung an. Dies wird daran deutlich, dass auf die oben zitierte Stelle der Satz folgt, ,,[d]ass sein Hier und Jetzt nicht die

3. - Wichtige philosophische Auseinandersetzungen dazu sind von Aristoteles, Augustinus, Bergson und Hegel gemacht worden.

4. - Vgl. Georg Wilhelm Friedrich Hegel Enzyklopädie. Bd. II. Hg. von Eva Moldenhauer, Karl Markus Michel, Frankfurt/Main, Suhrkamp, 1970, S. 41-55.

5. - Paul Virilio, Fluchtgeschwindigkeit, München/Wien, Hanser, 1996, S. 57.

6. - Thomas Glavinic, Das Leben der Wünsche, München, dtv, 2011, S. 289. 
einzige Wahrheit war. [Hervorhebung: FF] "7 Die enge Verknüpfung von Raum und Zeit wird in Glavinics Die Arbeit der Nacht erwähnt, wenn dort beschrieben wird, dass Zeit kein Nach- sondern ein Nebeneinander sei $^{8}$. Unter anderem an dieser Stelle wird klar, dass Gegenwartsliteratur trotz des 'spatial turn' in den Kulturwissenschaften noch immer in der Zeit eine fundamentale Kategorie, die es zu beschreiben gilt, sieht. Unter anderem kann dies in der gesellschaftlichen Entwicklung liegen, dass eine fortschreitende Technisierung mit einer Beschleunigung einher geht. Die Kritik an dieser Entwicklung ist wie schon geschrieben an dem populären Wort der Entschleunigung oder an Virilios Aufsätzen zu sehen.

Dennoch bleibt festzuhalten, dass bei Glavinic zur abstrakten philosophischen Perspektive auf Raum und Zeit die der subjektiven menschlichen Wahrnehmung hinzutritt. In einer solchen kann die Zeit selbstverständlich beschleunigt oder als ein Nebeneinander wahrgenommen werden.

In den Romanen Glavinics wird nicht die Zeit oder der Raum an sich thematisiert, sondern die menschliche Wahrnehmung eben dieser. So ist die philosophische Sicht auf Zeit, als ein Punkt im jeweiligen Jetzt zwischen dem Vergangenen und dem Kommenden, lediglich an wenigen Stellen explizit beschrieben ${ }^{9}$. Beispielsweise wird sie in der philosophischen Thematisierung von Zeit gerecht werdenden Allegorie des Seiltanzes bei Glavinic gewählt ${ }^{10}$.

In diesem Beitrag wird gezeigt, dass Zeit und Zeitwahrnehmung eine zentrale Funktion in der Roman-Trilogie haben. Dies ist bereits daran zu sehen, dass der Roman Das Leben der Wünsche und die beiden Erzählstränge in Das größere Wunder mit der Thematisierung von Zeit beginnen und Die Arbeit der Nacht und Das Leben der Wünsche mit Reflexionen über Zeit enden. Dass Zeit in Glavinics Texten zentral ist, zeigt sich auch darin, dass die erzählte Zeit in Das Leben der Wünsche nicht einer natürlichen Zeit folgend abläuft. Die Söhne des Witwers Jonas sind bei Anrufen zu verschiedenen Zeit-Momenten der erzählten Zeit immer sieben Tage im Urlaub und werden noch sieben weitere bleiben ${ }^{11}$. Es zeigt sich an dieser Stelle eine Relativität von Zeit und eine Ausrichtung an der subjektiv wahrgenommenen Zeit. Die tatsächlich verstreichende, beschleunigte Zeit wird der subjektiven

7. - Thomas Glavinic, Das Leben der Wünsche, München, dtv, 2011, S. 289.

8. - Vgl. Thomas Glavinic, Die Arbeit der Nacht, München, dtv, 2009 S. 394.

9. - Vgl. z.B. Thomas Glavinic, Das größere Wunder, München, Hanser, 2013, S. 7.

10. - Vgl. Thomas Glavinic, Die Arbeit der Nacht, München, dtv, 2009, S. 392.

11. - Vgl. Thomas Glavinic, Das Leben der Wünsche, München, dtv, 2011, u.a. S. 231 und S. 291. 
Zeitwahrnehmung des Erzählers untergeordnet und hat keine Bedeutung mehr. Diese subjektive Zeitwahrnehmung wird unter anderem deutlich als Jonas reflektiert: „Manche Erlebnisse, die Jahre zurücklagen, empfand er so gegenwärtig, daß er meinte, sie könnten keinesfalls zehn oder fünfzehn Jahre her sein, so nah und so wahrhaftig erschienen sie ihm"12.

Im folgenden soll zunächst auf die besonderen Implikationen von medialen Zeichen - wie zum Beispiel der Fotografie - und Zeit eingegangen werden, um daran anschließend die Versuche der medialen Entschleunigung, die der Protagonist Jonas an einigen Stellen umsetzt, zu erläutern. Die Wahrnehmung von Zeit, die in den Texten verhandelt wird, verbindet sich in allen drei Romanen mit der existenzialistischen Frage nach dem Dasein zum Tod. Das übergeordnete poetologische Projekt in der Roman-Trilogie ist jedoch, so die These meines Beitrags, eine Epiphanie der Augenblickswahrnehmung. Eine solche bewusste natürliche Wahrnehmung des Augenblicks ergo des Hier und Jetzt wird vom Protagonisten Jonas verfolgt beziehungsweise propagiert. Dies wird beispielsweise offenbar, wenn Jonas feststellt: „Er schlug wahrhaftig seine Zeit tot, und diese Erkenntnis erschreckte ihn über alle Maßen"13.

\section{Zeit und Medien bei Thomas Glavinic - unter besonderer Berücksichtigung der Romane: Die Arbeit der Nacht, Das Leben der Wünsche und Das größere Wunder}

Wie jedoch, so kann gefragt werden, entgeht man dem Totschlagen der Zeit? Eine Möglichkeit ist die mediale Archivierung von Zeit. Dies kann mittels verschiedener medialer Zeichenträger geschehen. Beispielsweise kann man Schrift beziehungsweise die Sprache verwenden ${ }^{14}$. Das Medium Sprache, als medialer Zeichenträger von Zeit wird vom Protagonist Jonas in allen drei Romanen genutzt. In Die Arbeit der Nacht schreibt sich der plötzlich gänzlich alleine auf der Welt seiende Jonas schriftliche Botschaften, um sich seiner eigenen Existenz innerhalb des Verstreichens von Zeit bewusst zu sein ${ }^{15}$. Diese $\mathrm{zu}$ verschiedenen Zeitpunkten an unterschiedlichen Orten hinterlassenen Zettel werden teilweise auch mit Datum und Uhrzeit versehen.

12. - Thomas Glavinic, Die Arbeit der Nacht, München, dtv, 2009, S. 144.

13. - Thomas Glavinic, Das größere Wunder, München, Hanser, 2013, S. 368.

14. - Diese Idee ist unter anderem in Lessings Schrift Laokoon zu finden.

15. - Vgl. Felix Forsbach Spur der Existenz. Die Hauptfigur in Glavinics, Die Arbeit der Nacht als medial vermittelte Existenz. In: Zwichen Alptraum und Glück. Thomas Glavinics Vermessungen der Gegenwart. Hg. von Andrea Bartl, Jörn Glasenapp, Iris Hermann. (=Poiesis. Standpunkte zur Gegenwartsliteratur, Bd. 10, Hg. von Friedhelm Marx), Göttingen, Wallstein, 2014, S. 132-146, hier: S. 133f. 
Diese Datierungen haben die Funktion, dem allein auf der Welt lebenden Jonas den Fortschritt der Zeit zu beweisen ${ }^{16}$. Neben Sprache dient auch der mediale Zeichenträger Film, insbesondere das Video, zur Archivierung von Zeit. In Die Arbeit der Nacht wird das Filmen exzessiv betrieben. Neben der nachhaltigen Archivierung dieser zeitlich und räumlich isolierten Ausschnitte aus der Wirklichkeit haben die Videos im Roman auch wieder die Funktion der Existenzbestätigung des Erzählers. Allerdings wird darüber hinaus auch deutlich, dass die Videoaufnahmen, die Jonas macht, die Funktion haben, Zeit (ein Hier und Jetzt) in der Zukunft wahrnehmbar zu machen. Jonas spricht medialen Zeichenträgern, im Gegensatz zu seiner Erinnerung, eine authentische Repräsentation der Vergangenheit $\mathrm{zu}^{17}$. Sowohl Videos, Schrift als auch Fotografien werden als Beweise gesehen, dass es die Momente beziehungsweise Zeit in der Vergangenheit tatsächlich gegeben hat. Eine besondere Authentizität im Bezug auf die Repräsentation von Zeitausschnitten wird den medialen Zeichenträgern Video und Fotografie zugeschrieben. Diese seien authentisch, weil sie nicht vom Menschen geschaffen werden, sondern maschinell erzeugt werden:

„Diese Aufnahme war ohne menschlichen Zeugen von einer Maschine gemacht worden. [...] Aber diese Bilder waren der Beweis, daß diese Minuten existiert hatten. Sie waren vorbei. Wenn er jetzt zur Brücke hinauflief, würde er eine andere Brücke, eine andere Zeit antreffen, als er hier sah. Doch es hatte sie gegeben“"18.

Auch die Fototheorie räumt der medialen Repräsentation in Fotografien eine erhöhte Authentizität ein. Dies kulminiert in Roland Barthes Noema des „Es-ist-so-gewesen“, das er in Die helle Kammer formuliert ${ }^{19}$. Die erhöhte Authentizität soll an dieser Stelle ebenso für die Videos gelten, weil sie von Jonas in ähnlicher Weise rezipiert und verwandt werden wie Fotografien. Durch das Medium Fotografie ist dem Menschen sogar die Möglichkeit gegeben, optisch unbewusste Momente wahrzunehmen und im Nachhinein genau zu betrachten. Diese Idee findet sich in Das Leben der Wünsche wieder, als Jonas nach der Beschreibung einiger kurzer Momentaufnahmen die Bedeutung von Fotos so beschreibt: „Ein Moment. Das war es. Deswegen machte er Fotos. Diese Sekunde hatte es gegeben, ohne dass sie jemand als solche

16. - Vgl.u.a. Thomas Glavinic, Die Arbeit der Nacht, München, dtv, 2009, S. 101.

17. - Vgl. u.a. ebd. S. 181.

18. - Ebd. S. 228.

19. - Vgl. Roland Barthes, Die helle Kammer. Bemerkungen zur Photographie, Frankfurt/Main, Suhrkamp, 1989, S. 103. 
wahrgenommen hatte“20. An dieser Stelle ist eine Analogie zu Walter Benjamins Idee des „optisch-unbewussten“ in Fotografien zu sehen ${ }^{21}$.

Außerdem wird ein zentraler Unterschied bezüglich der Wahrnehmung der Wirklichkeit in der natürlichen Wahrnehmung des Menschen und der technisch unterstützten Wahrnehmung offengelegt. Dieser Unterschied liegt in der Wirklichkeitsrezeption mit den menschlichen Sinnesorganen innerhalb der Wirklichkeit und der zeitlich versetzten Rezeption eines Wirklichkeitsausschnitts auf einem Foto. Denn auf der Fotografie kann ein kleiner zeitlich und räumlich isolierter Moment auf Dauer rezipierbar gemacht werden. Die Reflexion des Menschen über die Wirklichkeit ist immer nachzeitig. ,Jede Stelle, an die sie kamen, erreichte sein Bewusstsein, wenn überhaupt, erst Minuten oder gar Stunden später"22 formuliert Jonas in Das größere Wunder und betont die Fähigkeiten des Mediums Fotografie, indem er ,an seine Kamera [dachte], die hinten in seinem Rucksack steckte und mit der er dieses Panorama hätte festhalten können, um später zu verstehen, wo er gewesen war und was dies für ihn bedeutete" 23 . Auch das Medium Fotografie kann Momente nur nachzeitig rezipierbar machen. Setzt man - im Anschluss an Charles Sanders Peirce und Roland Barthes - die Indexikalität von Fotos voraus, so sind diese bezüglich der authentischen Repräsentation von Wirklichkeit besser als die direkte menschliche Sinneswahrnehmung. Außerdem haben Fotografien in Bezug auf die These dieses Beitrags einen großen Vorteil, sie können Zeitmomente isolierter festhalten. Denn eine Fotografie ist ein wahrnehmbarer Zeitausschnitt, der je nach Belichtungszeit weit unter einer Sekunde liegt. Somit kommt die Repräsentation der Zeit in der Fotografie dem philosophischen Ideal eines möglichst nah am Punkt sein, näher als die kontinuierlich fortlaufende Rezeption der Zeit durch die menschliche Sinneswahrnehmung.

In Fotos ist - zugespitzt gesagt - die Repräsentation eines JetztMoments und somit die Repräsentation von Zeit eher möglich als in jedem anderen medialen Zeichenträger. Diese Idee ist in Das Leben der Wünsche gleich im Anschluss an die oben schon zitierten Reflexionen Jonas' zu Fotografie angeschlossen. Denn es hatte die fotografisch festgehaltenen Sekunden tatsächlich gegeben und sie waren wie ,[e]ine Linie[, die...] aus einzelnen Punkten [bestand], die niemand sah. Die

20. - Thomas Glavinic, Das Leben der Wünsche, München, dtv, 2011, S. 43.

21. - Vgl. Walter Benjamin, Kleine Geschichte der Photographie, in: Walter Benjamin, Gesammelte Schriften, Bd. 2, Hg. von Rolf Tiedemann, Hermann Schweppenhäuser, Frankfurt/Main, 1977, S. 368-385, hier: S. 371.

22. - Thomas Glavinic, Das größere Wunder, München, Hanser, 2013, S. 482.

23. - Ebd. 


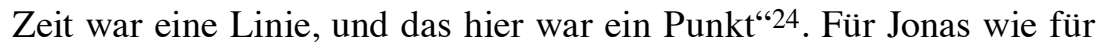
jeden Menschen gibt es beinahe unendlich viele dieser Jetzt-Momente oder Punkte. Es gibt wie Jonas sagt:

„Momente. Solche und solche, [...], schöne und miserable und gleichgültige. [...] Er stellte auf Aufnahme und hielt den Apparat nach links, drückte $a b$, nach rechts drückte ab. Er zoomte die Wiese heran und drückte ab. Das beste der drei Bilder war das erste. Viele Menschen, in ihrer Bewegung festgefroren. Eine Sekunde, gerade vorübergegangen, unbeobachtet geblieben, wertlos geworden, eine von Milliarden und Abermilliarden“ 25 .

Diese große Menge an isolierten Zeitmomenten ist für den Menschen nicht wahrnehmbar, geschweige denn zu verarbeiten. Im Grunde sei dies jedoch auch nicht wichtig, denn von den drei Fotos, die Jonas machte war eins das beste, somit könnte geschrieben werden, dass es genügen würde, genau diesen Zeitausschnitt auf dem besten Foto als Repräsentant einer größeren Zeiteinheit zu sehen.

Die Aufteilung großer Zeiträume auf einzelne isolierte JetztMomente, die Jonas auf Fotos festhält, ist auch in seinem Projekt zu sehen, ein Daumenkino seines Lebens zu erstellen. Dazu macht er mit seinem Fotoapparat seit vielen ,Jahren jeweils am ersten Tag eines jeden Monats [...] mit immer dem selben neutralen Hintergrund“ 26 ein Porträt von sich. Somit dient als Repräsentant seines Lebens, seiner Entwicklung und eventuell sogar seiner Existenz eine Reihe von isolierten Jetzt-Momenten. Auf Maries Frage, was dieses Daumenkino sei, antwortet Jonas folgerichtig:

„Einfach nur ich. Ich in der Zeit.

In welcher Zeit?

In mehr als zehn Jahren. Mitte zwanzig bis jetzt.“27

In allen drei Romanen der Trilogie spielen mediale Zeichen als Repräsentanten der gegenwärtigen Wirklichkeit und Vergangenheit eine zentrale Rolle. Jonas ist bezüglich der Mediennutzung ein Kind unserer beschleunigten Zeit. Es schreibt SMS, fotografiert, filmt et cetera. Doch alle medialen Zeichen, die er verwendet, haben auch die Funktion des Festhaltens von Zeit beziehungsweise von Jetzt-Momenten. Diese isolierten Zeitmomente können durch die den medialen Zeichenträgern inhärente Funktion der Medialisierung immer wieder rezipiert werden. Somit wird durch Medien eine nachzeitige Reflexion, Betrachtung und

24. - Thomas Glavinic, Das Leben der Wünsche, München, dtv, 2011, S. 43.

25. - Ebd. S. 97.

26. - Ebd. S. 43.

27. - Ebd. S. 284. 
Aufbewahrung von Jetzt-Momenten möglich. Wenngleich an dieser Stelle daran erinnert werden muss, dass sogar die zeitlich sehr isolierten Repräsentanten auf Fotos nur eine Annäherung an den philosophischen Begriff der Zeit in Form eines idealen Punktes auf einer Linie sein können.

Wegen der beschriebenen Möglichkeiten der medialen Zeichen im Allgemeinen und der Fotografie im Besonderen führt dies zu Jonas“ folgenden Überlegungen bezüglich des Vergehens von Zeit in Die Arbeit der Nacht:

„Beim Anblick des Fotos erinnerte er sich an seinen Gedanken, die fortschreitende Langsamkeit könne töten. Wenn dies stimmte, wenn man durch eine endlose Bewegung, die in der Bewegungslosigkeit endete, an der Ewigkeit anstreifte - überwog darin das Tröstende oder das Entsetzliche?"“28

Erinnert man an dieser Stelle an die anfangs wiedergegebenen Thesen zur allgemeinen Tendenz der Beschleunigung in unserer Gesellschaft, so kann Jonas ' Frage damit beantwortet werden, dass innerhalb des Lebens gewisse Bereiche nicht beschleunigt werden sollten, um eine tatsächliche menschliche Wahrnehmung von Jetzt-Momenten zu ermöglichen. Somit hat es etwas Tröstendes, dass es in der nachzeitigen Rezeption von Fotografien die Möglichkeit gibt, Jetzt-Momente verlangsamt und genauer wahrzunehmen. In dieser Vorstellung ist die Möglichkeit enthalten, dass es eine mediale Entschleunigung geben kann.

\section{Mediale „Entschleunigung“}

Als Beispiel für den Wunsch der Veränderung des natürlichen und eventuell beschleunigten Fortlaufens der Zeit kann das mehrfach bei Glavinic erwähnte Märchen gesehen werden, in dem ein Mädchen mittels einer Spule Zeit schneller oder langsamer ablaufen lassen kann ${ }^{29}$. Dieser Wunsch kann Jonas nicht erfüllt werden. Allerdings existiert die Möglichkeit Zeit, die medial repräsentiert wird, schneller oder langsamer ablaufen zu lassen. Dies vollzieht Jonas exzessiv mit den Videoaufnahmen in Die Arbeit der Nacht, die er sowohl in Zeitlupe als auch Zeitraffer anschaut ${ }^{30}$. Die Fähigkeit die Wirklichkeit wie einen Film verlangsamt oder beschleunigt wahrzunehmen, wird in den Romanen mehrfach beschrieben. Zeit beziehungsweise eher die Wahrnehmung ebendieser wird gedehnt oder beschleunigt ${ }^{31}$. Jonas

28. - Thomas Glavinic, Die Arbeit der Nacht, München, dtv, 2009 S. 90.

29. - Vgl. u.a. ebd. S. 127f.

30. - Vgl. ebd. S. 90f.

31. - Vgl. ebd. S. 49. 
fragt sich, ,[w]ie wird es sein, diese langsamen schnellen Sekunden zu erleben“. Außerdem enden sowohl Die Arbeit der Nacht, als auch Das Leben der Wünsche mit der Beschreibung eines Nahtoderlebnisses ${ }^{32}$. In diesen Beschreibungen wird stark über Zeit reflektiert und die Enden der Romane sind als eine Form der gedehnten Zeitwahrnehmung zu sehen. Es kann als Form des filmischen Schreibens bezeichnet werden, wobei dieses filmische Schreiben einer Zeitlupeneinstellung im Film gleicht. Als Beispiel für diese Zeitreflexion sollen einige der letzten Sätze des Romans Das Leben der Wünsche genügen: „Für eine Sekunde schien sich die Welt nicht weiterzubewegen. [...] Das Bild seiner Welt wackelte. Hielt für eine Sekunde an" ${ }^{33}$. Auch in Die Arbeit der Nacht wird Zeitwahrnehmung gedehnt: „Er hatte nicht gewußt, daß Zeit so zäh war“"34. Die natürliche Zeitwahrnehmung wird an dieser Stelle als unnatürlich beziehungsweise filmisch beschrieben. Dies ist auch zu erkennen, wenn Jonas denkt:

„Sein Leben schien bisweilen schneller abzulaufen, um urplötzlich zu stoppen und ihn etwas Entscheidendes behutsam erleben zu lassen. Bald darauf lebte er wieder wie vor einer Kamera, schnell, doch nicht schmerzlos“35.

Jedoch vergleicht Jonas an dieser Stelle die veränderte natürliche Wahrnehmung mit dem Dasein ,vor einer Kamera“. Die Wahrnehmung Jonas' in den Extremsituationen der Schlusskapitel der Romane Das Leben der Wünsche und Die Arbeit der Nacht gleicht sich an die mediale Zeitwahrnehmung an. Es kann sogar behauptet werden, dass die Wahrnehmung des Menschen Jonas medial wird ${ }^{36}$ :

„Eine Sekunde. Und abermals: eine Sekunde.

Ein Schnappschuss"37.

„Er wandte sich Marie zu. Sie betrachtete ihn offenbar schon länger.

Das Bild, das er sah, schien zu ruckeln wie eine DVD mit Kratzern.

32. - Es ist nicht eindeutig ob es sich tatsächlich um die Beschreibungen von den letzten Sekunden vor dem Tod oder ein Nahtoderlebnis handelt. An dieser Stelle solle keine Interpretation der recht offenen und uneindeutigen Romanenden geliefert werden, jedoch ist eindeutig, dass die Romane mit einer Reflexion über Zeit enden, was in diesem Beitrag das Zentrum der Untersuchung ist.

33. - Thomas Glavinic, Das Leben der Wünsche, München, dtv, 2011, S. 317.

34. - Thomas Glavinic, Die Arbeit der Nacht, München, dtv, 2009, S. 393.

35. - Thomas Glavinic, Das Leben der Wünsche, München, dtv, 2011, S. 123.

36. - Vgl. Felix Forsbach Spur der Existenz. Die Hauptfigur in Glavinics, Die Arbeit der Nacht als medial vermittelte Existenz. In: Zwischen Alptraum und Glück. Thomas Glavinics Vermessungen der Gegenwart. Hg. von Andrea Bartl, Jörn Glasenapp, Iris Hermann (=Poiesis. Standpunkte zur Gegenwartsliteratur, Bd. 10, Hg. von Friedhelm Marx), Göttingen, Wallstein, 2014, S. 132-146, hier: S. 143ff.

37. - Thomas Glavinic, Das Leben der Wünsche, München, dtv, 2011, S. 317. 
Eine Sekunde Standbild. Wirklichkeit und Zeit vereinigten sich wieder. Brachen abermals auseinander" 38 .

Die Wahrnehmung des Erzählers ist medialisiert. Darin ist sicherlich keine Zeit- oder Medienkritik Glavinics zu sehen. Vielmehr scheint es bei Glavinics Texten zentral zu sein, die Gegenwart zu beschreiben, und diese Gegenwart ist - folgt man beispielsweise Virilio - beschleunigt oder aber auch technisiert beziehungsweise medialisiert. Glavinics Texte verdeutlichen das Potenzial der Fiktion, Phantastik und Surrealität, das die exzessive Mediennutzung unserer Zeit bürgt. Wie bereits beschrieben ist das Thema des Todes und der menschlichen Existenz in den Texten Glavinics neben den Themen Zeit, Glück und Liebe omnipräsent. Daher soll im Folgenden kurz auf die Implikationen von Zeit im Bezug auf menschliche Existenz und den Tod eingegangen werden.

\section{Zeit und Existenz als Dasein zum Tod}

In allen drei Romanen Glavinics sind Beschreibungen von Nahtoderfahrungen zu finden. In Das größere Wunder zum Beispiel rast der adoleszente Jonas mit Freunden in einem Traktor mit extrem überhöhter Geschwindigkeit eine Straße herunter.

„Je schneller der Traktor den Berg hinabraste, desto tiefer versank Jonas in einem Gefühl vollkommener Leichtigkeit. Er fühlte sich schwerelos, geborgen und heiter. Es war, als müsste er nicht mehr atmen, um zu leben. Kein Gedanke störte seine Seligkeit, keine Erinnerung sucht ihn heim, er vermisste nichts und niemanden. Links und rechts der Straße flogen Bäume und Sträucher an ihm vorbei, und er war wie neu. Ewig stürzen. Das ist das Glück ${ }^{39 “}$.

Innerhalb dieser Beschreibungen, die hier als Nahtoderfahrungen gedeutet werden, erscheint Zeit in einer Art Verlangsamung wahrnehmbar zu sein. Jonas kann in diesen Momenten, so scheint es, einzelne Hier und Jetzt Momente tatsächlich wahrnehmen. Hinzu tritt die absolute Fokussierung auf das Hier und Jetzt, denn „keine Erinnerungen suchten ihn heim“40. Ein weiteres Beispiel für diese Fokussierung auf

38. - Ebd. S. 318.

39. - Thomas Glavinic, Das größere Wunder, München, Hanser, 2013, S. 151.

40. - Ein weiteres interessantes Untersuchungsfeld in der Roman-Trilogie, das aufgrund des Umfangs an dieser Stelle nicht geleistet werden kann, ist die Beschreibung von Erinnerung bei Glavinic. Sehr häufig erinnert sich der Protagonist aufgrund von Gerüchen an Vergangenes. Beziehungsweise Erinnerung wird sehr nah an den Geruchssinn und Düfte gebunden. Genauso, wie Erinnerung in den Romanen Glavinics in Form von Erinnerungsbildern beschrieben wird. Sowohl zu der Verbindung Geruch und Erinnerung als auch Bild und Erinnerung sind zahlreiche Abhandlungen in der Forschungsliteratur zu finden. Vgl. z.B. Aleida Assmann, Erinnerungsräume. Formen 
das Hier und Jetzt ist der womögliche Suizid von Jonas als er in Die Arbeit der Nacht vom Wiener Fernsehturm springt:

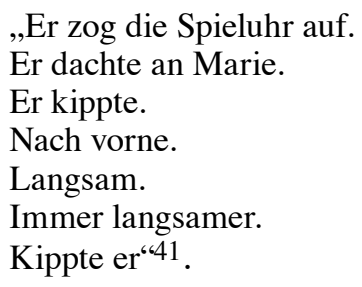

Im Anschluss an diesen Sprung in die Tiefe werden zahlreiche Reflexionen über Zeit in die Wahrnehmung Jonas' innerhalb der letzten Sekunden seines Sturzes eingebunden. So stellt er fest, ,[d] as war die Zeit, das war die Persönlichkeit in der Zeit." ${ }^{2} 2$ Wenngleich sich die Gedanken und Wahrnehmungen dieser vermeintlich letzten Sekunden unterscheiden, kulminieren sie dennoch in einer übergeordneten Kategorie: Dem Glück.

Zieht man an dieser Stelle nun Martin Heideggers Überlegungen aus Sein und Zeit hinzu, drängt sich angesichts der Nahtoderfahrungen Heideggers Vorstellung der „cura“ auf. Dahinter verbirgt sich die Idee, dass das gesamte Dasein in seiner Suche nach dem Sein immer in der Sorge lebe zu sterben. Daraus resultiert auch die pessimistische Vorstellung eines Daseins zu Tod. Es werde, so Heidegger, das gesamte Leben als eine Zeit, die unabänderlich auf ihr Ende zu steuere gesehen. Somit wird das Leben durch den Tod bestimmt. ${ }^{43}$ Die Problematik bei Heideggers Untersuchungen ist unter anderem darin $\mathrm{zu}$ sehen, dass der Mensch im Grunde das Sein nicht erreichen kann, sondern im Dasein verharrt. Eine ähnliche unerreichbare Vorstellung von etwas Unerreichbarem ist bei Glavinic im Glück und der Liebe zu sehen. Die scheinbar authentischen Glücks- oder Liebesmomente tauchen in den Romanen meist in Verbindung mit existenziell bedrohlichen Situationen auf.

An dieser Stelle soll nicht soweit gegangen werden im Glück und der Liebe Heideggers Sein zu deuten. Jedoch ist evident, dass die Verbindung von Tod mit Glück und Liebe in Glavinics Texten oft einher geht.

Wie zuvor im Anschluss an die Zitate schon festgestellt, spielt die Wahrnehmung von Zeit in diesen existenziellen Momenten für Jonas eine übergeordnete Rolle. Deswegen soll im folgenden letzten Punkt

und Wandlungen des kulturellen Gedächtnisses, München, Beck, 2010, S.218-241.

41. - Thomas Glavinic, Die Arbeit der Nacht, München, dtv, 2009, S. $391 f$.

42. - Ebd. S. 392.

43. - Vgl. Martin Heidegger, Sein und Zeit, Tübingen, Max Niemeyer, 2006, S. 191-196. 
auf die Verbindung von den positiven und im Dasein unerreichbaren Momenten des Glücks und der Liebe eingegangen werden.

\section{Das Dasein wahrnehmen: Epiphanie des Augenblicks}

In den Schlusskapiteln von Das Leben der Wünsche ist ein Nahtoderlebnis in Form einer riesigen an Tsunamis erinnernden Welle, die auf Marie und Jonas zukommt, zu sehen ${ }^{44}$. Im Angesicht dieser Welle schien sich die Welt für den Erzähler ,[f]ür eine Sekunde [...] nicht weiterzubewegen" 45 . In der Literaturwissenschaft wird das Motiv des Wassers und Meeres häufig in Verbindung mit Zeit und Ewigkeit gedeutet. Diese Tradition kann auch hier aufgegriffen werden, denn im Gegensatz zur Ewigkeit des Wassers reflektiert das zum Tode bestimmte menschliche Subjekt sein Leben und die Zeit. Wobei im Moment des nahenden Todes vor allem kurze Zeiteinheiten eine wichtige Rolle spielen. Jonas nimmt diesen Moment als Moment und Hier und Jetzt wahr.

„Eine Sekunde. Und abermals: eine Sekunde.

Ein Schnappschuss" ${ }^{46}$.

Im Schnappschuss kann wieder eine Form der Medialisierung der Wahrnehmung gesehen werden, genauso jedoch ist in Bezug auf diese Textstelle zu betonen, dass es sich um die natürliche menschliche Wahrnehmung eines Jetzt-Moments handelt. Diese Sekunde erscheint in der Erzählung gedehnt zu sein, denn es werden viele Bilder, Erinnerungen, Wahrnehmungen geschildert, die alle innerhalb von nur „eine[r] Sekunde. Eine[r] einzige[n] Sekunde. Eine[r] zufällige[n], lange[n], alte[n] Sekunde, hier und jetzt, jetzt und einst, eine[r] von Milliarden und Abermilliarden" ${ }^{47}$ geschildert werden.

Innerhalb dieser Sekunde verspürt Jonas das Gefühl des Glücks und der Liebe ${ }^{48}$. Ebenso wie in Das Leben der Wünsche empfindet Jonas im Moment des Sprungs vom Wiener Fernsehturm in Die Arbeit der Nacht innerhalb der als gedehnte Zeit wahrnehmbaren Schilderungen seiner Reflexionen und Erinnerungen das Glück und die Liebe als zeitlich und generell übergeordnete Kategorien und womöglich als das Sein des Menschen ${ }^{49}$. Neben diesen Epiphanien des Augenblicks und

44. - Vgl. zur Metaphorik des Wassers auch Thomas Glavinic, Das größere Wunder, München, Hanser, 2013, S. 214.

45. - Thomas Glavinic, Das Leben der Wünsche, München, dtv, 2011, S. 317.

46. - Ebd.

47. - Ebd. S. 319.

48. - Vgl. Ebd. S. 318.

49. - Vgl. Thomas Glavinic, Die Arbeit der Nacht, München, dtv, 2009, S. 395. 
der Wahrnehmung des Augenblicks, die mit Glück und Liebe assoziiert sind, wird auch im Schriftbild bei Glavinic eine Hervorhebung des Hier und Jetzt betrieben. Beispielsweise wenn der Erzähler sagt:

„Dieser Stein war ein Unikat. Ein zweiter wie dieser, den er gerade jetzt in der Hand hielt, existierte nicht“50.

So wird auch im Schriftsatz eine Betonung und Epiphanie der Jetzt-Momente betrieben. Es ist für Jonas wichtig, einzelne Momente bewusst wahrzunehmen, wie an zahlreichen Stellen deutlich wird. Unter anderem, wenn er sagt: „Er bemühte sich nach Kräften, die Situation in seine innere Galerie ewiger Augenblicke aufzunehmen“ 51 . Oder: „Er konzentrierte sich. Mit aller Kraft versuchte er sich im Hier und Jetzt festzuhalten" 52 . Dennoch ist sich Jonas der Flüchtigkeit der Augenblicke bewusst und er resümiert: „Es war ein Jetzt. Nun Teil eines Damals“53. Es ist eindeutig, dass Augenblicke also nicht dauerhaft gemacht werden können. Insbesondere die individuelle Momente des Glücks und der Liebe können nicht dauerhaft gemacht werden, sie werden in Augenblicken, Jetzt-Momenten wahrgenommen. Diese Epiphanie des Augenblicks und der Augenblickwahrnehmung mit der Verbindung der Gefühle von Glück und Liebe können auch in medialen Zeichenträgern nicht dauerhaft gemacht werden. Und auch die Veränderung der natürlichen menschlichen Wahrnehmung scheint nicht auszureichen, wie die Geschichte über den Mönch, der sich seine Augenlider abschnitt, zeigt, um die gesamte Wirklichkeit wahrzunehmen ${ }^{54}$.

Das poetologische Projekt Thomas Glavinics ist es, Momente des Glücks und der Liebe als besondere Augenblicke im Leben von Menschen darzustellen. Diese Augenblicke sollen, will man Glück und Liebe wahrnehmen, in entschleunigter und bewusster Wahrnehmung aufgenommen werden, denn sie sind flüchtig und können nicht dauerhaft gemacht werden.

Die Roman-Trilogie endet folgerichtig in Das größere Wunder mit einer langen Aneinanderreihung von Momentwahrnehmungen Jonas;, die durch die womöglich ewige Liebe von ihm und Marie ausgelöst sind. Die bewusste Wahrnehmung und das Fühlen der Liebe werden als

50. - Thomas Glavinic, Die Arbeit der Nacht, München, dtv, 2009, S. 389. Ein weiteres Beispiel für die Isolation von Momenten im Schriftsatz ist in Thomas Glavinic, Das Leben der Wünsche, München, dtv, 2011, S. 313 und Thomas Glavinic, Das größere Wunder, München, Hanser, 2013, S. 326, 383, 396, 486 zu sehen.

51. - Thomas Glavinic, Das größere Wunder, München, Hanser, 2013, S. 400.

52. - Thomas Glavinic, Das Leben der Wünsche, München, dtv, 2011, S. 102.

53. - Thomas Glavinic, Das größere Wunder, München, Hanser, 2013, S. 443.

54. - Vgl. Thomas Glavinic, Das Leben der Wünsche, München, dtv, 2011, S. 180. 
Augenblick dargestellt, wohl der wichtigste Augenblick eines Menschen. Jonas ist sich auch an dieser Stelle dessen bewusst, dass es sich um einen epiphanen flüchtigen Augenblick handelt.

„Sie legte sich zu ihm, und er dachte noch: Niemals vergessen, niemals vergessen, aber manches ist zu groß und zu wertvoll, um es behalten zu können, und man kann es niemals berichten, auch sich selber nicht. Es war in der Sekunde weg, in der es gekommen war, es gehörte ihnen nur in genau der Sekunde, in der es passierte“" 55 .

55. - Thomas Glavinic, Das größere Wunder, München, Hanser, 2013, S. 521. 